\title{
ON MAXIMAL TRANSITIVE SUBTOURNAMENTS
}

\author{
by J. W. MOON† \\ (Received 11th March 1971)
}

\section{Introduction}

A tournament $T_{n}$ consists of a finite set of nodes $1,2, \ldots, n$ such that each pair of distinct nodes $i$ and $j$ is joined by exactly one of the arcs $i j$ or $j i$. If the arc $i j$ is in $T_{n}$ we say that $i$ beats $j$ or $j$ loses to $i$ and write $i \rightarrow j$. If each node of a subtournament $A$ beats each node of a subtournament $B$ we write $A \rightarrow B$ and let $A+B$ denote the tournament determined by the nodes of $A$ and $B$.

A tournament $T_{n}$ is transitive if its nodes can be labelled in such a way that $i \rightarrow j$ if and only if $i>j$; in this case we call node $n$ the top node. A transitive subtournament of a tournament $T_{n}$ is maximal if it is not a proper subtournament of any other transitive subtournament of $T_{n}$. Let $f(n)$ denote the maximum number of maximal transitive subtournaments a tournament $T_{n}$ can have; we find by inspection, for example, that $f(1)=f(2)=1$ and $f(3)=f(4)=3$. Our object here is to prove the following result.

Theorem. If $n=5 m+r$, where $m \geqq 1$ and $0 \leqq r \leqq 4$, then

$$
c_{r} 7^{m-1} \leqq f(n) \leqq(1 \cdot 717)^{n}
$$

where $c_{0}=7, c_{1}=9, c_{2}=15, c_{3}=19$, and $c_{4}=31$.

Corollary. If $\theta=\lim _{n \rightarrow \infty}(f(n))^{1 / n}$, then $\theta$ exists and $1 \cdot 4757 \leqq \theta \leqq 1 \cdot 717$.

\section{A lower bound for $f(n)$}

A tournament $T_{n}$ is strong if it cannot be expressed as $T_{n}=A+B$ for some nonempty tournaments $A$ and $B$. If $T_{n}$ is not strong it has a unique expression of the type $T_{n}=A+B+\ldots+K$ where the non-empty tournaments $A, B, \ldots, K$ all are strong; if this is the case, then $f\left(T_{n}\right)=f(A) f(B) \ldots f(K)$ where $f(X)$ denotes the number of maximal transitive subtournaments in the tournament $X$. It follows, therefore, that if $a+b=n$ then

$$
f(n) \geqq f(a) f(b) \text {. }
$$

If $T_{n}$ is any tournament with $n$ nodes, let $T_{n+2}$ denote the tournament obtained by adjoining two nodes $p$ and $q$ to $T_{n}$ such that $p \rightarrow T_{n}, T_{n} \rightarrow q$, and $q \rightarrow p$. It is not difficult to see that $f\left(T_{n+2}\right)=2 f\left(T_{n}\right)+1$; consequently,

$$
f(n+2) \geqq 2 f(n)+1 \text {. }
$$

$\dagger$ On leave from the University of Alberta. 
Since $f(3)=3$, it follows that $f(5) \geqq 7, f(7) \geqq 15$, and $f(9) \geqq 31$; furthermore, $f(6) \geqq(f(3))^{2}=9$, by (1), whence $f(8) \geqq 19$ by (2).

Let us now suppose that $n=5 m+r$ where $m \geqq 2$ and $0 \leqq r \leqq 4$. Then

$$
f(n) \geqq f(5(m-1)) f(5+r) \geqq c_{r}(f(5))^{m-1} \geqq c_{r} 7^{m-1},
$$

by (1) and the results in the preceding paragraph. We remark that the existence of the limit in the corollary follows from inequality (1) and a well-known result on sub-additive functions (2, Problem 98, pp. 17, 171).

\section{An upper bound for $f(n)$ : a special case}

We shall prove that $f\left(T_{n}\right) \leqq \beta^{n}$, where $\beta=1 \cdot 717$, by induction on $n$. The inequality certainly holds when $1 \leqq n \leqq 4$ and we may restrict our attention to strong tournaments $T_{n}$ in view of the observation made earlier.

The score of a node $i$ in a tournament is the number $s_{i}$ of nodes that $i$ beats. If $x$ is the top node of any maximal transitive subtournament $M$ of a tournament $T_{n}$, let $N$ denote the tournament obtained from $M$ by deleting $x$ ( $M$ must have at least two nodes when $n \geqq 2$ ). It is easy to verify that $N$ is a maximal transitive subtournament of the tournament determined by the $s_{x}$ nodes of $T_{n}$ that lose to $x$; thus $x$ is the top node of at most $f\left(s_{x}\right)$ maximal transitive subtournaments of $T_{n}$. It follows, therefore, that if $\left(s_{1}, \ldots, s_{n}\right)$ denotes the score sequence of a tournament $T_{n}$, then

$$
f\left(T_{n}\right) \leqq \sum_{i=1}^{n} f\left(s_{i}\right)
$$

If $T_{n}$ is strong then $s_{i} \leqq n-2$ for every node $i$. In this section we treat the case where there exists a node $p$ in $T_{n}$ such that $s_{p}=n-2$. Let $q$ denote the unique node of $T_{n}$ that beats $p$. If two nodes of $T_{n}$ have score $n-2$, then we may take $p$ and $q$ to be these nodes. If three nodes had score $n-2$ then these nodes would beat all the remaining nodes when $n>3$ and $T_{n}$ would not be strong. Thus we may suppose that $s_{i} \leqq n-3$ for any node $i$ of $T_{n}$ other than $p$ or $q$. Let $T_{a}$ and $T_{b}$ denote the subtournaments determined by those nodes of $T_{n}$ other than $p$ that beat $q$ and lose to $q$, respectively. Since $s_{q} \leqq n-2$ it must be that $a \geqq 1$ and $b \leqq n-3$.

Node $q$ is the only node that beats $p$. It follows that every maximal transitive subtournament of $T_{n}$ that does not contain $q$ must certainly contain $p$. It is not difficult to see that there are at most $f(n-2)$ such subtournaments. A maximal transitive subtournament that contains both $p$ and $q$ cannot contain any nodes of $T_{a}$, for it would not be transitive otherwise. There are at most $f(b)$ such subtournaments (we adopt the convention that $f(0)=1$ ).

We now consider those maximal transitive subtournaments of $T_{n}$ that contain $q$ but not $p$. There are certainly no more than $f(n-1)$ such subtournaments in general. We can obtain a sharper bound when $a \leqq 2$ by observing that the top node of such subtournaments must belong to $T_{a}$; if this were not the case then they would contain no nodes of $T_{a}$ and node $p$ could be adjoined 
without destroying the transitivity properly. Hence, there are at most af $(n-3)$ such subtournaments.

We may suppose that $f(m)<\beta^{m}$ if $m<n$ and that $f(m)$ is an increasing function. It follows, therefore, that if $T_{n}$ is strong and has a node of score $n-2$, then

$$
f\left(T_{n}\right) \leqq \begin{cases}2 f(n-3)+f(n-2) & \leqq(2+\beta) \beta^{n-3}, \\ f(n-4)+2 f(n-3)+f(n-2) \leqq(1+\beta)^{2} \beta^{n-4}, \\ f(n-5)+f(n-2)+f(n-1) \leqq\left(1+\beta^{3}+\beta^{4}\right) \beta^{n-5},\end{cases}
$$

according as $b=n-3, b=n-4$, or $b \leqq n-5$. Each of the last three bounds is smaller than $\beta^{n}$ when $\beta=1.717$ and a similar argument shows that $f\left(T_{n}\right) \leqq \beta^{n}$ when $T_{n}$ is strong and has a node of score 1 . (Notice that the first three bounds imply that $f\left(T_{5}\right) \leqq 7$ when $T_{5}$ has a node of score 1 or 3 ; we shall use this inequality in the next section.)

\section{An upper bound for $f(n)$ : the general case}

Let $T_{n}$ denote a strong tournament with no nodes of score 1 or $n-2$; if the nodes of $T_{n}$ are labelled so that the sequence $s=\left(s_{1}, \ldots, s_{n}\right)$ of scores is nondecreasing, then it follows from our assumptions that

$$
\begin{gathered}
2 \leqq s_{1} \leqq \ldots \leqq s_{n} \leqq n-3, \\
\sum_{i=1}^{k} s_{i} \geqq\left(\begin{array}{l}
k \\
2
\end{array}\right)+1, \text { for } 1 \leqq k \leqq n-1, \text { and } \\
\sum_{i=1}^{n} s_{i}=\left(\begin{array}{l}
n \\
2
\end{array}\right) .
\end{gathered}
$$

We remark that for any sequence $s$ satisfying these conditions there exists at least one tournament with score sequence $s$; this is a consequence of a theorem due to Landau (see $(1 ;$ p. 61$)$ ).

We first treat the cases where $5 \leqq n \leqq 8$. It follows from inequality (3) that $f\left(T_{n}\right) \leqq n f(n-3)$ for tournaments $T_{n}$ whose scores satisfy conditions (4)-(6). Consequently, $f\left(T_{5}\right)<5 \cdot 1, f\left(T_{6}\right) \leqq 6 \cdot 3, f\left(T_{7}\right) \leqq 7 \cdot 3$, and $f\left(T_{8}\right) \leqq 8 \cdot 7$ for such tournaments when $5 \leqq n \leqq 8$. All these bounds are less than $\beta^{n}$ for the appropriate values of $n$, so we may now suppose that $n \geqq 9$ and that $f(m) \leqq \beta^{m}$ for $m<n$.

If $n \geqq 9$, let $S_{n}$ denote the set of all sequences $s=\left(s_{1}, \ldots, s_{n}\right)$ of $n$ integers that satisfy conditions (4)-(6). Let $s^{*}$ denote the sequence $(2,2,2,2,4,6,6,6,6)$ or $(2,2,2,2,3,5,6, \ldots, n-6, n-4, n-3, n-3, n-3, n-3)$ according as $n=9$ or $n \geqq 10 ;$ it is easy to verify that $s^{*} \in S_{n}$. If $g(x)=\beta^{x}$ let

$$
G(s)=g\left(s_{1}\right)+\ldots+g\left(s_{n}\right)
$$

for any $s$ in $S_{n}$. We shall prove the following result in the next section. 
Lemma. If $s \in S_{n}$ where $n \geqq 9$, then $G(s) \leqq G\left(s^{*}\right)$. that

It follows from inequality (3), the induction hypothesis, and the lemma,

$$
f\left(T_{9}\right) \leqq G(s) \leqq G\left(s^{*}\right)=4 \beta^{2}+\beta^{4}+4 \beta^{6} \leqq \beta^{9}
$$

and, in general, that

$$
\begin{aligned}
f\left(T_{n}\right) & \leqq G(s) \leqq G\left(s^{*}\right)=4 \beta^{2}+\beta^{3}+\left(\beta^{n-5}-\beta^{5}\right) /(\beta-1)+\beta^{n-4}+4 \beta^{n-3} \\
& \leqq \beta^{n-5} /(\beta-1)+\beta^{n-4}+4 \beta^{n-3} \leqq \beta^{n}
\end{aligned}
$$

when $\beta=1 \cdot 717$. This will suffice to complete the proof of the theorem. (We remark that it is easy to show that if $T_{n}$ is strong and $n \geqq 3$ then the minimum value $f\left(T_{n}\right)$ can have is 3 .)

\section{Proof of the lemma}

Let $s$ denote any sequence in $S_{n}$ such that $G(s)=\max \left\{G(t): t \in S_{n}\right\}$; such a sequence certainly exists. We shall prove the lemma by establishing a series of assertions (the only property of the function $g(x)$ that we use is that it is strictly convex).

Assertion 1. If two consecutive elements of $s$ are equal, they must equal 2 or $n-3$.

Suppose there exist integers $u$ and $v$, where $1 \leqq u<v \leqq n$, such that

$$
2<s_{u}=\ldots=s_{v}<n-3 ;
$$

we may suppose that $s_{u-1}<s_{u}$ if $u>1$ and $s_{v}<s_{v+1}$ if $v<n$. Let $r=\left(r_{1}, \ldots, r_{n}\right)$ denote the sequence that differs from $s$ only in that $r_{u}=s_{u}-1$ and $r_{v}=s_{v}+1$. The sequence $r$ certainly satisfies conditions (4) and (6); it satisfies condition (5) as well unless there exists an integer $k$, where $u \leqq k<v$, such that

$$
\sum_{i=1}^{k} s_{i}=\left(\begin{array}{l}
k \\
2
\end{array}\right)+1
$$

If $k=1$ then $s_{1}=1$ and if $k=n-1$ then $s_{n}=n-2$ by (6); both these alternatives contradict condition (4) so we may suppose $2 \leqq k \leqq n-2$.

It follows from (7) and condition (5) that

$$
s_{k}=\sum_{i=1}^{k} s_{i}-\sum_{i=1}^{k-1} s_{i} \leqq\left(\begin{array}{l}
k \\
2
\end{array}\right)+1-\left(\begin{array}{c}
k-1 \\
2
\end{array}\right)-1=k-1 .
$$

Furthermore, $s_{k+1}=s_{k}$ so

$$
\sum_{i=1}^{k+1} s_{i} \leqq\left(\begin{array}{l}
k \\
2
\end{array}\right)+1+(k-1)=\left(\begin{array}{c}
k+1 \\
2
\end{array}\right) \text {. }
$$

This contradicts condition (5) so it must be that the assumption that equation (7) holds is incorrect. Consequently, if assertion 1 does not hold then the sequence $r$ is in $S_{n}$. Since $g$ is a strictly convex function it follows that

$$
G(r)-G(s)=(g(x+1)-g(x))-(g(x)-g(x-1))>0
$$


where $x=s_{u}=s_{v}$. This contradicts the definition of $s$ so it must be that assertion 1 does in fact hold.

Assertion 2. The sequence $s$ must start with between two and four 2's and end with between two and four $(n-3)$ 's.

There are at most $(n-4)-2=n-6 s_{i}$ 's between 3 and $n-4$, inclusive, by Assertion 1 ; thus there are at least six $s_{i}$ 's equal to 2 or $n-3$. It is easy to verify that $s$ would not satisfy condition (5) if it started with more than four 2's or ended with more than four $(n-3)$ 's. Assertion 2 now follows.

Assertion 3. The sequence $s$ must start with four 2's and end with four $(n-3)$ 's.

If $s$ does not start with four 2's then $2=s_{1}=\ldots=s_{a-1}<s_{a}$ where $a=3$ or 4 , by Assertion 2. Now $a+1<n-3$, since $n \geqq 9$, so $s_{a}<s_{a+1}<s_{a+2}$, by Assertions 1 and 2 . Let $r=\left(r_{1}, \ldots, r_{n}\right)$ denote the sequence that differs from $s$ only in that $r_{a}=s_{a}-1$ and $r_{a+1}=s_{a+1}+1$. It is easy to verify that $r$ is in $S_{n}$ in this case. However,

$$
G(r)-G(s)=(g(y+1)-g(y))-(g(x)-g(x-1))>0
$$

where $x=s_{a}<s_{a+1}=y$. This contradiction implies that $s$ must start with four 2 's and the last part of the assertion can be proved in a similar way.

Assertion 4. $s=s^{*}$.

It follows from Assertions 1 and 3 that the middle $n-8$ elements of $s$ consist of $n-8$ of the $n-6$ numbers $3,4, \ldots, n-4$. Condition (6) implies that if $h$ is one of the missing numbers then $n-1-h$ is the other, where we may suppose that $3 \leqq h<\frac{1}{2}(n-1)$. If $h \geqq 5$ then $s$ does not satisfy condition (5) when $k=6$ so $h=3$ or 4 . When $n=9$ the only possibility is that $h=3$; when $n \geqq 10$ both values are possible but the function $G$ has the larger value for the sequence corresponding to $h=4$. It follows, therefore, that $s=s^{*}$ and the lemma is proved.

The preparation of this paper was assisted by a grant from the National Research Council of Canada.

\section{REFERENCES}

(1) J. W. Moon, Topics on Tournaments (Holt, New York, 1968).

(2) G. Pólya u. G. Szegö, Aufgaben und Lehrsätze aus der Analysis, I (Springer, Berlin, 1925).

Mathematical Institutf

OXFORD 